\title{
Article
}

\section{Towards Health Equality: Optimizing Hierarchical Healthcare Facilities towards Maximal Accessibility Equality in Shenzhen, China}

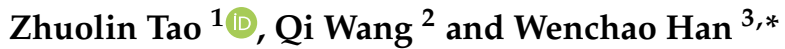 \\ 1 Faculty of Geographical Science, Beijing Normal University, No. 19, Xinjiekouwai Ave., Beijing 100875, China; \\ taozhuolin@bnu.edu.cn \\ 2 Proficiency Skill Appraisal and Guidance Center of Natural Resources Ministry, Beijing 100830, China; \\ 201221170050@mail.bnu.edu.cn \\ 3 Chinese Research Academy of Environmental Sciences, Beijing 100012, China \\ * Correspondence: 201631490013@mail.bnu.edu.cn
}

Citation: Tao, Z.; Wang, Q.; Han, W. Towards Health Equality: Optimizing Hierarchical Healthcare Facilities towards Maximal Accessibility Equality in Shenzhen, China. Appl. Sci. 2021, 11, 10282. https://doi.org/ 10.3390/app112110282

Academic Editor: Jianbo Gao

Received: 27 September 2021

Accepted: 27 October 2021

Published: 2 November 2021

Publisher's Note: MDPI stays neutral with regard to jurisdictional claims in published maps and institutional affiliations.

Copyright: (c) 2021 by the authors. Licensee MDPI, Basel, Switzerland. This article is an open access article distributed under the terms and conditions of the Creative Commons Attribution (CC BY) license (https:// creativecommons.org/licenses/by/ $4.0 /)$.

\begin{abstract}
Equal accessibility to healthcare services is essential to the achievement of health equality. Recent studies have made important progresses in leveraging GIS-based location-allocation models to optimize the equality of healthcare accessibility, but have overlooked the hierarchical nature of facilities. This study developed a hierarchical maximal accessibility equality model for optimizing hierarchical healthcare facilities. The model aims to maximize the equality of healthcare facilities, which is quantified as the variance of the accessibility to facilities at each level. It also accounts for different catchment area sizes of, and distance friction effects for hierarchical facilities. To make the optimization more realistic, it can also simultaneously consider both existing and new facilities that can be located anywhere. The model was operationalized in a case study of Shenzhen, China. Empirical results indicate that the optimal healthcare facility allocation based on the model provided more equal accessibility than the status quo. Compared to the current distribution, the accessibility equality of tertiary and secondary healthcare facilities in optimal solutions can be improved by $40 \%$ and $38 \%$, respectively. Both newly added facilities and adjustments of existing facilities are needed to achieve equal healthcare accessibility. Furthermore, the optimization results are quite different for facilities at different levels, which highlights the feasibility and value of the proposed hierarchical maximal accessibility equality model. This study provides transferable methods for the equality-oriented optimization and planning of hierarchical facilities.
\end{abstract}

Keywords: health equality; spatial optimization; hierarchical healthcare facilities; maximal accessibility equality; 2SFCA

\section{Introduction}

Healthcare services are widely regarded as one of the essential public services that affect residents' health and well-being. Efficient and equal provision of healthcare services to the population is always at the center of the governance and planning of healthy cities [1]. From the spatial perspective, the distribution of healthcare facilities directly influences the accessibility of residents to healthcare services and the utilization of healthcare services, which in turn impact their respective health outcomes [2-4]. Accessibility is a multidimensional concept that is related to both spatial and non-spatial factors $[5,6]$. The concept of spatial accessibility is adopted in this study, which measures how easily and how many opportunities can be reached by residents from different locations [7]. Ensuring essential and equal accessibility to healthcare services is a key target of the Sustainable Development Goal proposed by the United Nations [8,9]. In China, both the central and municipal governments have set up strategies to promote the equalization of healthcare services $[10,11]$. The worldwide outbreak of the COVID-19 pandemic and its far-reaching 
impacts have significantly highlighted health and safety issues and the rational planning of healthcare resources [12,13].

However, the distribution of healthcare services decided in the traditional manner often do not provide equal accessibility to all [3,14-16]. There are significant disparities in healthcare accessibility across different locations or different socio-economic groups (e.g., natives vs. immigrants, high-income vs. local income, and the elderly vs. the young) [17-19], which has important spatial/social equity implications [20]. In Shenzhen, China, the study area, significant inequality in healthcare accessibility has also been revealed by existing studies $[16,21]$. The irrational distribution of healthcare facilities and inequality of accessibility make a strong call for the optimization of healthcare facilities.

Academia from fields such as public health and geography have paid increased attention to the optimization of healthcare facilities. A series of optimization models have been developed, which are usually known as the location-allocation models [22,23]. Typically, these models set up one or more objective functions and a set of constraints [24]. Efficiency and equality are the most important objectives for allocating public facilities to different sites [25]. However, most of existing studies focus on the efficiency objectives such as minimizing the numbers or cost of facilities, maximizing the coverage of facilities, and minimizing the travel cost between consumers and facilities [26,27]. By contrast, little attention has been paid to equality of facilities distribution in location-allocation studies, partially due to the difficulty in modelling and optimizing equality [4].

Recently, an innovative stream of studies has considered spatial/accessibility equality in the location-allocation analysis [28-31]. The maximal accessibility equality (MAE) model developed by Wang and Tang [28] is a novel and helpful method for researchers and practitioners who are interested in improving the equality in demanders' accessibility to public services (e.g., healthcare services). However, the development and implementation of the MAE model are still confined to single-level facilities. Comparatively little attention has been paid to the equality optimization of hierarchical (or multi-level) healthcare facilities. As existing studies [32-34] have demonstrated, spatial analysis of hierarchical facilities should account for more characteristics such as various service scopes, frictions of distance, and transport modes. Therefore, the existent MAE model is not suitable for analyzing accessibility to hierarchical facilities. Although hierarchical location-allocation problems have been studied for decades [35-37], few have addressed the equality issue or incorporated spatial accessibility into location-allocation analysis. There are still gaps in terms of simultaneously considering the hierarchical nature and accessibility equality optimization of healthcare facilities.

This study's contributions are threefold. First, it develops a hierarchical maximal accessibility equality (HMAE) model, which is hierarchy-sensitive and can act as a useful tool in the equality-oriented spatial optimization of hierarchical healthcare facilities or other hierarchical facilities. Second, this study provides a method that simultaneously accounts for both existing fixed facility locations and newly added locations that are flexible in the location-allocation analysis. This can make the optimized solution more feasible because the fixed resources/stocks of existing facilities are considered in the optimization. Third, online map application programming interface (API) is introduced to improve the accuracy of estimated travel time in location-allocation analysis. The proposed model maximizes the equality of spatial accessibility to healthcare facilities by minimizing the variation in accessibility across all locations. This is achieved by both adding new facilities and reallocating the resources at existing facilities. The method is valuable for the implementation of equality-oriented healthcare planning and policymaking. Shenzhen, which is one of the first cities to have highlighted the policy goal to achieve equality in healthcare services in China, was selected as the study area to demonstrate the feasibility and usefulness of the proposed model. 


\section{Literature Review}

\subsection{Classic Location-Allocation Models}

Serving as a tool for people to analyze and optimize locations of facilities, locationallocation models have been present for more than five decades [22]. There are a set of such models that are termed as the classic location-allocation models [24,26]. In addition to the p-median model, the classic models also include the maximal covering location model, the location set covering model, and the p-center model. The covering models deal with the coverage of demanders (usually represented by discrete and aggregate locations) within a certain radius of each facility [38]. The maximal covering location model aims to maximize the coverage on the basis of a certain number of facilities [27], whereas the location set covering model is designated to achieve full coverage using the least number of facilities. The p-center model is different from the above models, aiming to minimize the maximal distance from each demand nodes to its nearest facility. It is also known as the minimax problem [24]. To some extent, the p-center model considers equity issues by improving the situation of the remotest demanders.

The classic models are tailored to approach various policy objectives and have engendered numerous applications [22,24]. The classic models have also been extended and improved in other instances, e.g., the gravity p-median model that incorporates a gravity rule into the p-median model [39], making them applicable in more complicated real-world contexts. The classic models, however, are faced with several drawbacks [4]. First, they fail to explicitly address the equity issue. Most of the existing location-allocation models only address efficiency-oriented objectives. Second, the assumptions of the spatial interaction between demanders and facilities in these models are relatively simple. Most notably, few have employed realistic accessibility measurement when considering how demanders reach facilities.

\subsection{Hierarchical Location-Allocation Problems}

Researchers have developed location-allocation models for hierarchical facilities. Hierarchical facilities are a type of facility that consist of multi-level facilities, facilities at each level that provide (totally or partially) different functions of service within different territories [36,40]. A healthcare facility is a typical type of hierarchical facility [32].

From a modelling perspective, hierarchical facilities can be classified according to their flow patterns, service varieties, spatial configurations, and optimization objectives [36,37]. Flow pattern is about the organization and delivery of services among different levels of facilities. The single-flow pattern assumes demanders at each node are serviced in facilities at the lowest-level, then transferred to facilities at higher levels, if necessary. The multiflow pattern indicates that demanders can be allocated to facilities at any level. Service varieties determine whether the functions at a lower level can also be supplied at higher levels. According to spatial configurations, the service scopes of facilities at a lower level should be in accord with those at higher levels. The optimization objectives of hierarchical location-allocation models are mainly built on the basis of the classic models described above, e.g., the hierarchical p-median model, hierarchical maximal covering model, and hierarchical location set covering model $[35,41,42]$. Therefore, these models focus on the efficiency of facility configurations and more or less overlook equity/equality issues. In addition, most hierarchical location-allocation models also fail to address the complex interactions between the demand and supply. In other words, the two drawbacks of the classic models previously pointed out by Wang [4] persist in hierarchical models.

\subsection{The Maximal Accessibility Equality Model}

Aiming to address the equality issue, Wang and Tang [28] initiated a novel locationallocation model, termed the "maximal accessibility equality" (MAE) model. The MAE model quantifies the equality dimension of facilities' spatial configuration as the sum of squares of differences in the accessibility to different facilities. The optimal configuration would minimize the disparity in the accessibility to facilities [28]. The objective function 
of this problem represents how spatial equality is understood and quantified. Solving the problem thus addresses the policy concerns over equality in public services. The MAE model can be expressed and solved as a quadratic programming problem.

Tao et al. [29] applied the maximal equality model to analyze optimal configuration of residential facilities and introduced the particle swarm optimization heuristic algorithm to solve the model. Another study tried to extend it by selecting newly added facility locations rather than reallocating resources at existing or given locations [43]. Two studies further introduced a two-step procedure, with the first step to optimize locations of facilities, while the second step to optimize the respective sizes of the facilities [30,44]. Dai et al. [31] incorporated a random allocation mechanism into the MAE model to optimize educational opportunities. To date, however, few have paid attention to the equality optimization of hierarchical facilities. To achieve this goal, the MAE model needs to be extended to account for hierarchical nature of facilities.

Note that a few recent studies have made efforts to model the spatial accessibility to hierarchical healthcare facilities [32-34]. These studies adapted the spatial accessibility measurements, e.g., the two-step floating catchment area (2SFCA) method, in order to account for the hierarchical characteristics of facilities, including variable service scopes, different distance frictions, and different transport modes for facilities at various levels. Although these studies fail to improve and optimize the accessibility to hierarchical facilities, the above advancements in modelling accessibility to hierarchical facilities can help to develop a hierarchical version of MAE model. The current study makes further efforts to combine the measurement of accessibility to hierarchical facilities and the MAE model that optimizes the equality of accessibility.

\section{Data and Methods}

\subsection{Study Area and Data}

Shenzhen was chosen for the empirical analysis. It was selected because existing studies have revealed hierarchical features in healthcare facilities and in accessibility to these facilities in Shenzhen [32]. Shenzhen is one of the special economic zones and megacities in China. It is located in the Pearl River Delta region in China. Shenzhen has undergone rapid socio-economic development in the last four decades since China's reform and opening in 1978. By 2018, Shenzhen has 11 million permanent residents and 1997 square kilometers of land area. It is composed of 10 administrative districts, 55 subdistricts (or jiedao in Chinese), and 771 communities (or Shequ in Chinese) (see Figure 1). In Shenzhen, like in other Chinese cities, public healthcare facilities play a predominant role in the provision of healthcare services to the residents. According to the "Medical Regulations of Shenzhen Special Economic Zone" issued by Shenzhen's municipal government in 2016, healthcare facilities in the city are organized as a three-level system. They are the tertiary, secondary, and primary healthcare facilities from the top to the bottom. The primary facilities consist of the community health service centers (CHSCs) serving the city's 771 communities. The secondary and tertiary facilities serve the 10 districts and the city, respectively. The current hierarchical healthcare system in Shenzhen is not well established, wherein the referral system between various levels has not been formulated, and patients are free to choose healthcare facilities at various levels [16].

The data used in this study comprised three types:

(1) community-level population counts;

(2) point-level healthcare facilities with attribute information such as names, hierarchy, number of physicians, and addresses;

(3) the travel time between community centroids to healthcare facilities. 


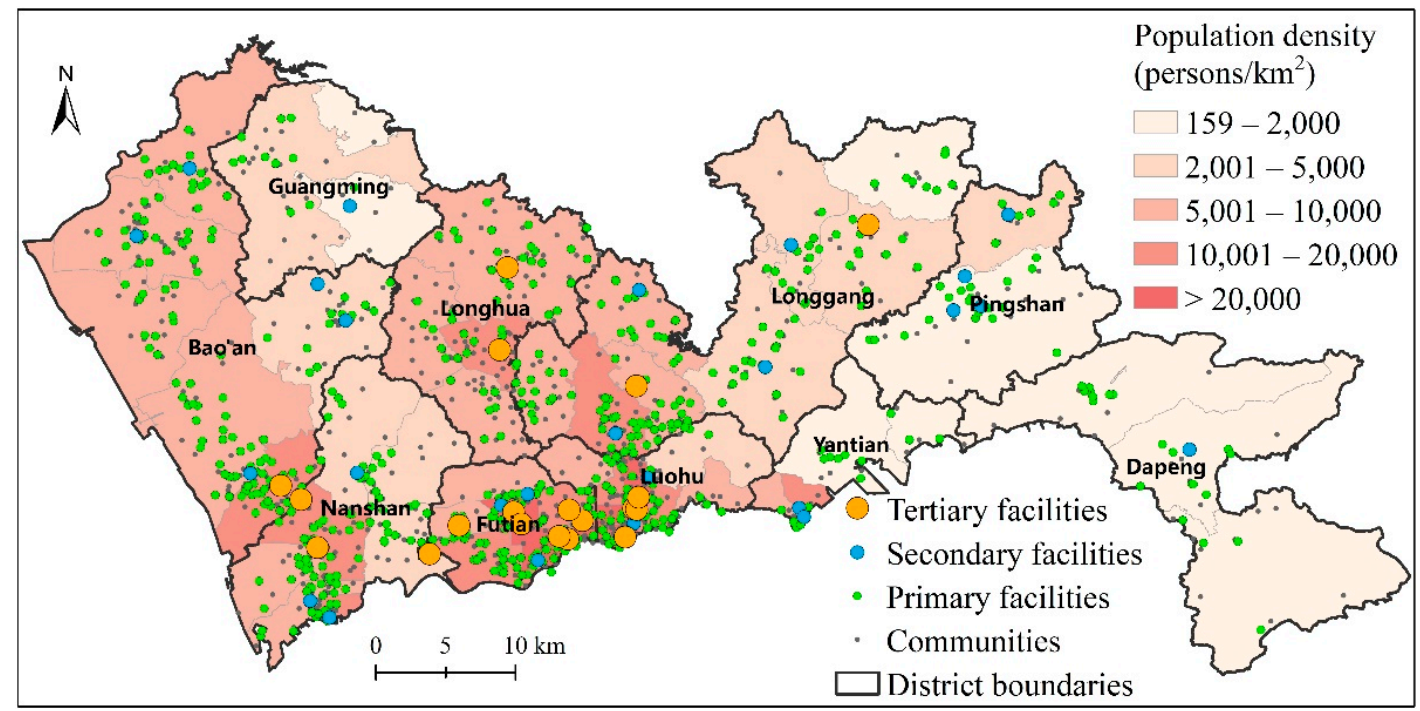

Figure 1. Location and distribution of population and healthcare facilities in Shenzhen.

The population counts are from the sixth population census of China, which was conducted in 2010 and is the most up to date of its kind available to the public. Centroids of communities and their geospatial information such as longitude and latitude coordinates are obtained via the geocoding API of Baidu Maps. The average population size of each community is 13,500. Each community is treated as a demand node in our ensuing analyses.

Detailed information concerning all the three levels' facilities is available at the official website of Shenzhen Municipal Health Commission [45]. As of December 2018, there were 19 tertiary healthcare facilities, 35 secondary healthcare facilities, and 612 primary healthcare facilities in Shenzhen. The average numbers of physicians in these facilities were 422, 182, and 6 physicians, respectively (Table 1).

Table 1. Basic statistics of the hierarchical healthcare facilities in Shenzhen.

\begin{tabular}{cccc}
\hline Facility Levels & Number of Facilities & Total Physicians & Average Physicians \\
\hline Primary facilities & 612 & 3672 & 6 \\
Secondary facilities & 35 & 6377 & 182 \\
Tertiary facilities & 19 & 8012 & 474 \\
\hline
\end{tabular}

The primary facilities are the largest in quantity and the smallest in the average number of physicians. They are widely distributed in all districts in Shenzhen (Figure 1). The primary facilities provide incredibly wide-ranging functions and service qualities that they usually become the first choice of patients residing in proximity. Therefore, the maximal equality model may be not applicable to these facilities. Furthermore, existing studies have found that the spatial accessibility to primary hospitals is relatively equal in Shenzhen [32]. Given this, only the tertiary and secondary facilities were considered in this study. In brief, tertiary and secondary healthcare facilities are different mainly in three aspects. First, tertiary facilities usually provide more complicated and higher-level services than secondary facilities. Second, tertiary facilities usually have larger coverage areas than secondary facilities, which can be reflected by the different catchment sizes in the model. Third, the average size (number of physicians) of tertiary facilities is significantly larger than that of secondary facilities.

The measurement of spatial accessibility relies on the travel times between demand nodes and different facility locations. Following existing studies $[16,21]$, the travel times are estimated using the driving navigation API of Baidu Map [46], the most popular online map in China. The estimation is based on real-world transportation network, historical traffic congestion information, and the local driving rules. The departure time of these trips 
are assumed to be between 10 a.m. and 5 p.m. on weekdays. This is done to avoid extreme travel times during peak hours.

\subsection{The Hierarchical Maximal Accessibility Equality (HMAE) Model}

The maximal equality model was developed by Wang and Tang [28]. It aims to achieve equal accessibility by minimizing the variation in the spatial accessibility to healthcare facilities from different demand nodes. In this study, the original MAE model was further adapted into a hierarchical version. The objective function can be expressed as Equation (1).

$$
\text { minimize } \sum_{i}^{m} P_{i}\left(A_{i}^{l}-\frac{\sum_{i}^{m} P_{i} A_{i}^{l}}{\sum_{i}^{m} P_{i}}\right)^{2}
$$

where $A_{i}^{l}$ is the spatial accessibility at demand node $i$ to facilities at level $l, P_{i}$ is the population, and $m$ is the number of demand nodes (i.e., communities in this study). The function means the population-weighted sum of the difference between the accessibility at each node and the population-weighted average accessibility. In the original study, spatial accessibility is calculated using the 2SFCA method. The generalized form of 2SFCA can be written as Equation (2).

$$
A_{i}^{l}=\sum_{j}^{n} \frac{S_{j}^{l} f\left(d_{i j}\right)}{\sum_{k}^{m} P_{k} f\left(d_{k j}\right)}
$$

where $S_{j}^{l}$ is the supply size (amount of physicians in this study) at candidate level-l facility location $j, n$ is the amount of candidate facility locations, $d_{i j}$ is the travel cost (e.g., travel time or distance) between each demand node and each candidate facility location, and $f$ is a function that describes the distance friction effect. In this study, the Gaussian-based 2SFCA method is adopted to measure spatial accessibility, which is advocated by existing studies on measuring healthcare accessibility [16]. The model takes a Gaussian distance friction function that is suitable for hierarchical facilities, which can be expressed as Equation (3):

$$
f\left(d_{i j}\right)=\left\{\begin{array}{c}
\frac{e^{-1 / 2 \times\left(d_{i j} / D_{l}\right)^{2}}-e^{-1 / 2}}{1-e^{-1 / 2}}, d_{i j} \leq D_{l} \\
0, d_{i j}>D_{l}
\end{array}\right.
$$

where $D_{l}$ is the catchment area size, i.e., the radius of service scope, of candidate facility at level $l$. Note that in the traditional spatial accessibility and maximal equality optimization studies, the catchment area is the same for all facilities. When applied to hierarchical facilities, however, this setting is inappropriate. Following existing studies on the spatial accessibility to hierarchical healthcare facilities [32], we assigned different catchment sizes for facilities at different levels. Generally, the catchment size is larger for facilities at a higher level. Furthermore, on the basis of the Gaussian function, a larger catchment size also means a weaker distance friction for higher levels, which is another important characteristic of hierarchical healthcare facilities [32]. Note that the HMAE model in this study intends to maximize the equality of accessibility to healthcare facilities for each level independently. The reason for this setting is that the current hierarchical healthcare system in Shenzhen is a multi-flow and nested hierarchical system, where facilities at various levels provide service to residents independently [16].

\subsection{Implementation of the HMAE Model}

The process of spatial optimization is to determine the optimal value of the decision variable that can optimize the objective function for each facility level (tertiary and secondary) independently. Figure 2 summarizes the procedures in this study. The decision variable of the maximal equality model is the supply size $S_{j}$ at each candidate location. It can be zero, which means no facility is located at the location, or any positive value. Therefore, the selection of candidate locations is crucial for spatial optimization. In existing 
studies, there are two ways to select candidate locations. The first way is to set up candidate locations without consideration of existing facilities. The candidate locations can be the centroids or random locations within administrative or census units, or evenly distributed locations across the study area. The second way, by contrast, aims to rearrange the supply of existing facilities. In other words, the locations of existing facilities are used as candidate locations. The advantage of the second way is that the existing resources can be accounted for, making it more cost-efficient and realistic for policy decision making.

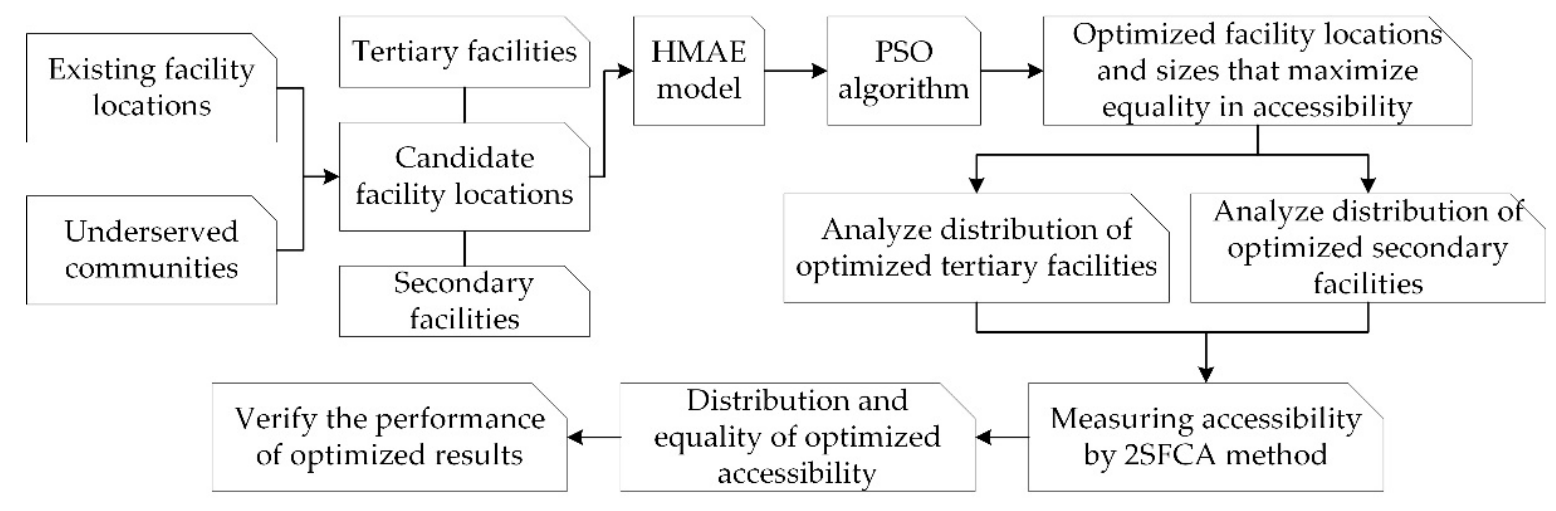

Figure 2. The framework of the procedures in this study.

In this study, the selection of candidate locations was based on the existing facility locations. Furthermore, considering that the existing facility locations might not be enough to provide coverage to all demand nodes within given catchment areas, we also accounted for the possibility that new facility locations may be needed. Despite the existing facility locations, we examined whether there are demand nodes that are located outside the catchment areas of all existing facilities. If yes, additional candidate locations were be selected from these underserved demand nodes. In sum, candidate locations for locating facilities consisted of two parts, i.e., existing facilities and underserved demand nodes. The selected candidate locations are described below.

This study introduces online map API to improve the estimation of travel time from patients to facilities. Specifically, the driving and transit navigation APIs provided by Baidu Map, a leading online map provider in China, were utilized to estimate travel time by driving or by public transit, respectively. Online map API can provide more accurate and reliable estimates of travel time on the basis of the frequently updated transport network, navigation rules, transit schedule, and traffic status $[16,47]$.

A few studies have demonstrated that various transport modes should be considered in accessibility analysis such that heterogenous demand of different socio-economic groups can be reflected $[16,34,48]$. This study considered two transport modes, i.e., private car and public transit. The latter includes both regular buses and subway and inter-mode transfers. Following Tao and Cheng [19], travel times by the two modes were combined on the basis of modal shares. Modal shares of private car, bus, and subway at the district level were collected from the Shenzhen 2016 Travel Survey.

As for hierarchical healthcare facilities, the catchment sizes should vary across different levels. Following existing studies [19,21], we determined the catchment sizes on the basis of the exceptional breakpoint of the distribution of the travel time from each demand node to the closest existing facility. The threshold was determined so that most demand nodes were within the catchment areas of existing facilities. Only a few extreme demand nodes that were extremely far away from existing facilities were excluded from the catchment areas. As a result, the catchment sizes for tertiary and secondary facilities were 70 and 40 min of travel times, respectively (see Figure 3). 


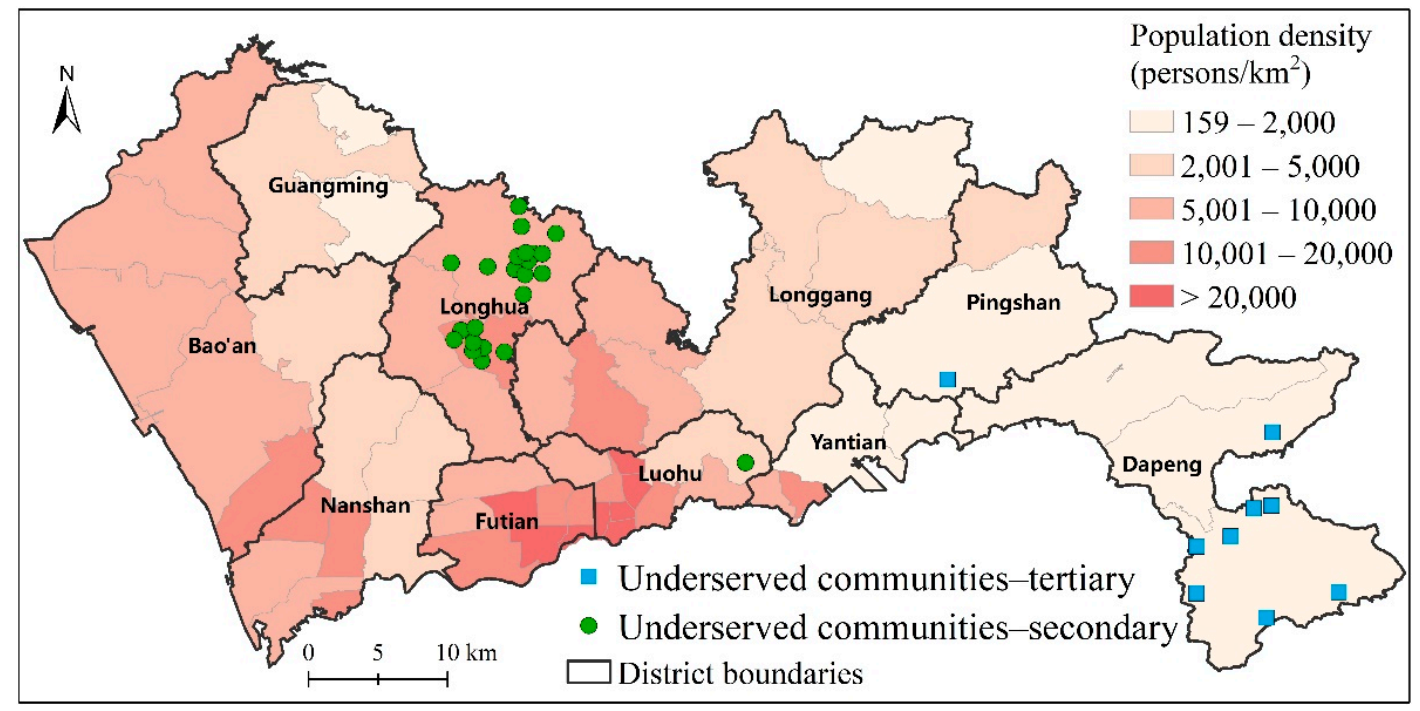

Figure 3. Underserved demand nodes of existing healthcare facilities.

In the next step, the travel times from each demand node to the closest existing tertiary and secondary facilities were calculated on the basis of the travel time matrix. If the minimum travel time between a demand node and tertiary facilities was larger than the catchment size, it could be considered to be an underserved demand node of tertiary facilities. The same procedure was executed for secondary facilities. The distribution of underserved demand nodes (communities) is shown in Figure 3.

The underserved demand nodes of tertiary facilities are concentrated in the east part of Shenzhen, one in Pingshan District, and the others in Dapeng District. Population density is relatively low in these areas. Therefore, one new candidate location was added in each district. Furthermore, even though all demand nodes in the west part of Shenzhen (i.e., Bao'an and Guangming districts) are covered by existing tertiary facilities, most of these demand nodes are quite far away from existing tertiary facilities. The closest tertiary facility is in the southernmost area of Bao'an District. Therefore, two new candidate locations were added in the northern Bao'an and Guangming Districts. The underserved demand nodes of secondary facilities are mainly concentrated in Longhua district, where moderate population density presents. There is another underserved demand node in Luohu District. Although Luohu is one of the central districts, population density in this subdistrict is relatively low, where the highest mountain, Wutong mountain, is located. Therefore, new candidate locations were only selected in Longhua District, on the basis of the distribution pattern of underserved demand nodes. Taken together, as shown in Figure 3, there are 23 and 39 candidate locations for tertiary facilities and secondary facilities, respectively. The average supply size of each facility was set as the same with existing facilities at each level. The total numbers of physicians at the two levels were found to be 9700 and 7100, respectively. Table 2 summarizes the setting of all parameters.

Table 2. Parameters at each level.

\begin{tabular}{ccccc}
\hline Facility Level & Catchment Size & $\begin{array}{c}\text { Number of Underserved } \\
\text { Communities }\end{array}$ & $\begin{array}{c}\text { Number of Candidate } \\
\text { Locations }\end{array}$ & Total Physicians \\
\hline Secondary facilities & $40 \mathrm{~min}$ & 23 & 39 & 7100 \\
Tertiary facilities & $70 \mathrm{~min}$ & 9 & 23 & 9700 \\
\hline
\end{tabular}

Following existing studies [29,49], the HMAE model was solved by using the particle swarm optimization (PSO) algorithm developed by Kenned and Eberhart [50]. PSO specifies a fitness function to evaluate the performance of each possible solution, which is represented as the total accessibility difference calculated by Equation (1). It provides an 
efficient approach to solving optimization problems by simultaneously considering a wide range of possible solutions and moving towards the optimal solution in an evolutionary manner. Each solution is termed as a particle, which consists of the facility sizes at all candidate locations. In each iteration, the performance of current solutions is evaluated by the fitness function and compared with the previous iteration. If the current solution generates better performance, it would be retained and the solutions in the next iteration would be determined on the basis of the trend. These solutions are expected to converge to a global optimal solution after a certain number of iterations.

PSO was first introduced by Tao et al. [29] into the maximal equality model. PSO can be operationalized with a toolbox in MATLAB developed by Birge [51]. The implementation of PSO is required for set-up of a few parameters, among which range of $X$ (i.e., the size of facility) and dimension of particles are related to the specific case. Dimension of particles was set as the number of candidate locations, i.e., 39 and 23 for tertiary and secondary facilities, respectively. Range of $X$ is defined by upper bound and lower bound. The lower bound was set as 0 . The upper bound was set as two times of the size of the largest existing facility, i.e., 1500 and 800 for tertiary and secondary facilities, respectively. Other parameters were determined on the basis of the work of Tao et al. [29] and the default parameters given by the manual of the toolbox.

\section{Results}

\subsection{Optimal Distribution of Tertiary Healthcare Facilities}

In addition to the existing 19 tertiary facilities, four new facility locations were added in areas that are quite far away from existing facilities. The newly added locations are located in Bao'an, Guangming, Pingshan, and Dapeng Districts. The optimal sizes of these candidate locations, both the existing facilities and newly added locations, were determined by using the hierarchical maximal equality model, aiming to minimize the variation in the spatial accessibility to facilities for all demand nodes.

The results are shown in Figure 4. The optimized facilities were classified into small-, middle-, and large-sized facilities (corresponding to facilities with 100-300, 300-800, or 800-1200 physicians, respectively) on the basis of the natural-breaks method. The ratios of three types of facilities were $48 \%, 39 \%$, and $13 \%$, respectively. Small- and middlesized facilities are dominant. There are only three large-sized tertiary facilities, which are respectively located in Nanshan, Bao'an and Guangming Districts. By contrast, the tertiary facilities in Futian and Luohu Districts, which are regarded as the core of Shenzhen, are middle- or small-sized. However, a relatively large number of existing tertiary facilities are concentrated in Futian and Luohu Districts. The tertiary facilities in Longhua, Longgang, and Pingshan Districts, where the distribution of facilities is relatively dispersed, are mainly middle-sized. The only tertiary facility in Dapeng District, which is newly added, is smallsized, due to the low population density in Dapeng and the surrounding areas. Generally, the optimized distribution of tertiary healthcare facilities presents a pattern in that facilities in the central areas are densely distributed but small-to-middle-sized, while facilities in the peripheral areas are middle-to-large-sized but dispersedly distributed.

The differences between optimized sizes and actual sizes of existing tertiary facilities were also calculated. This can help determine which adjustments of existing facilities are needed to achieve the optimal distribution, which is useful for decision making. As shown in Figure 5, the differences were significant, indicating that large adjustments are needed to materialize healthcare accessibility equality. In other words, the distribution of existing tertiary facilities is poorly performed in terms of providing equal healthcare accessibility.

Existing tertiary facilities that require positive size adjustments are mainly located in Nanshan, Bao'an, Longgang, and Longhua districts, while most facilities in Luohu and Futian districts need to be cut down in size. The pattern reveals that to achieve equal accessibility, more healthcare resources need to be allocated in the peripheral areas. Note that in the optimized distribution, some downsized ("negative adjustment") facilities may be close to upsized ("positive adjustment") facilities, e.g., the example marked by a yellow 
box in Figure 5. In such cases, the positive and negative adjustments close to each other can be counteracted. By doing so, many costs of adjustments can be saved, but with only negligible impacts on resources distribution and healthcare accessibility.

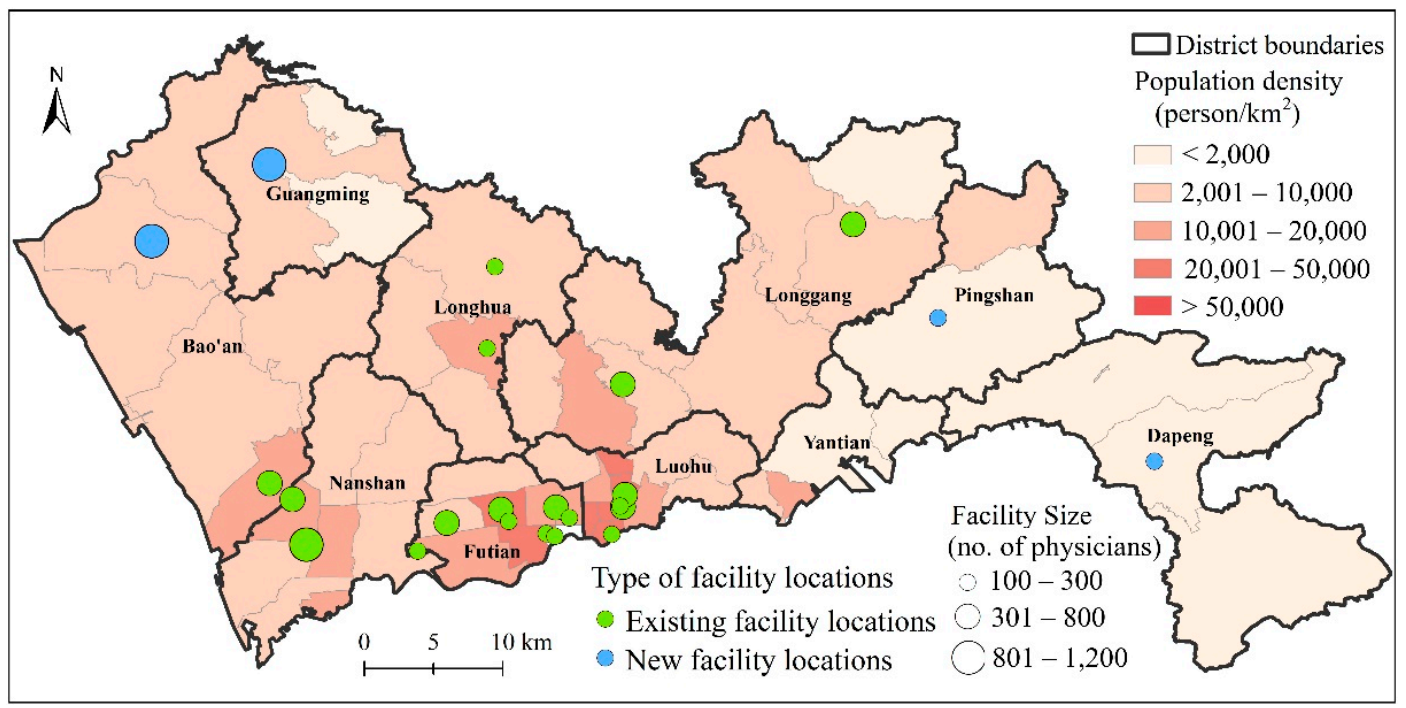

Figure 4. Optimized distribution of tertiary healthcare facilities.

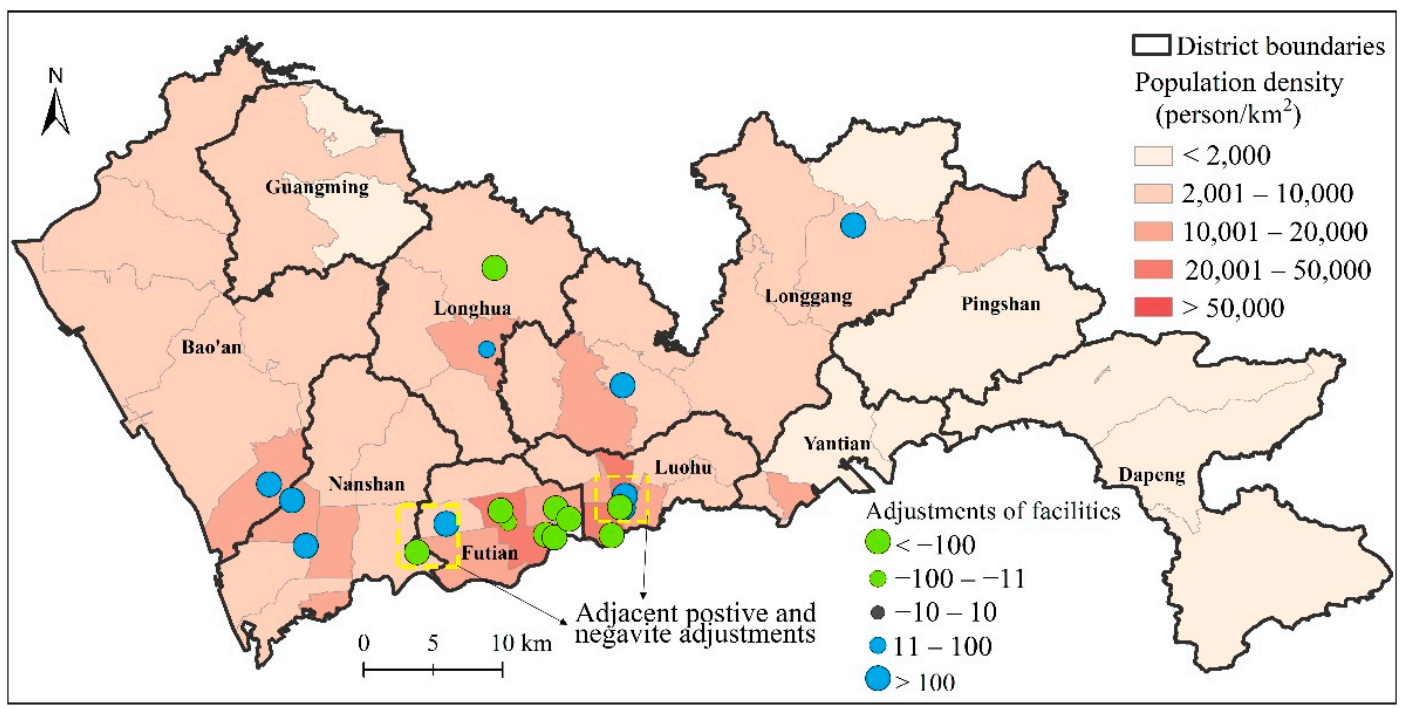

Figure 5. Size adjustments of existing tertiary healthcare facilities according to optimization.

\subsection{Optimal Distribution of Secondary Healthcare Facilities}

Similarly, the optimized secondary healthcare facilities are classified according to their sizes using the natural breaks method. The sizes of small-, middle-, and large-sized secondary facilities were less than 100, 100-300, and 300-600 physicians, respectively. The ratios of facility amount for three types were $46 \%, 36 \%$, and $18 \%$. The number of small-sized secondary facilities was the largest. As shown in Figure 6, the distribution of each type of secondary facilities is relatively even in most districts. In Pingshan, Dapeng, and Yantian districts, however, optimized secondary facilities were found to be relatively small in size. Large-sized secondary facilities with more than 300 physicians are relatively evenly distributed, which can cover moderate-to-high population density (higher than 10,000 persons $/ \mathrm{km}^{2}$ ) areas within a relatively small distance. 


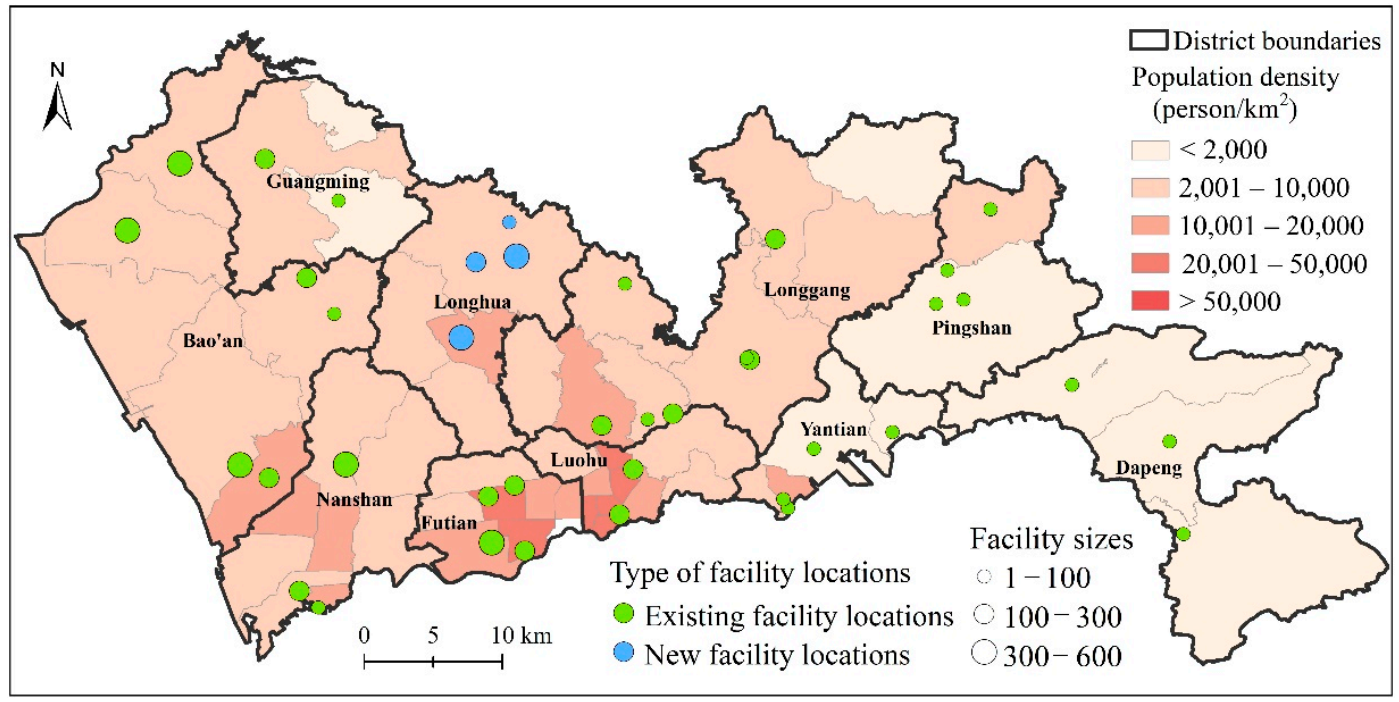

Figure 6. Optimized distribution of secondary healthcare facilities.

The needed size adjustments of existing secondary facilities to achieve equal accessibility are shown in Figure 7. Facilities that require positive size adjustments are mainly located in areas with relatively high population density. This indicates that the distribution of existing secondary facilities may fail to match the distribution of the demand, which can result in poor and unequal healthcare accessibility. There are also situations where negative and positive adjustment are close to each other, e.g., the areas marked by yellow boxes in Futian and southern Longgang districts. Counteracting these inverse size adjustments can make the optimized solution more economically feasible with negligible impacts on healthcare accessibility.

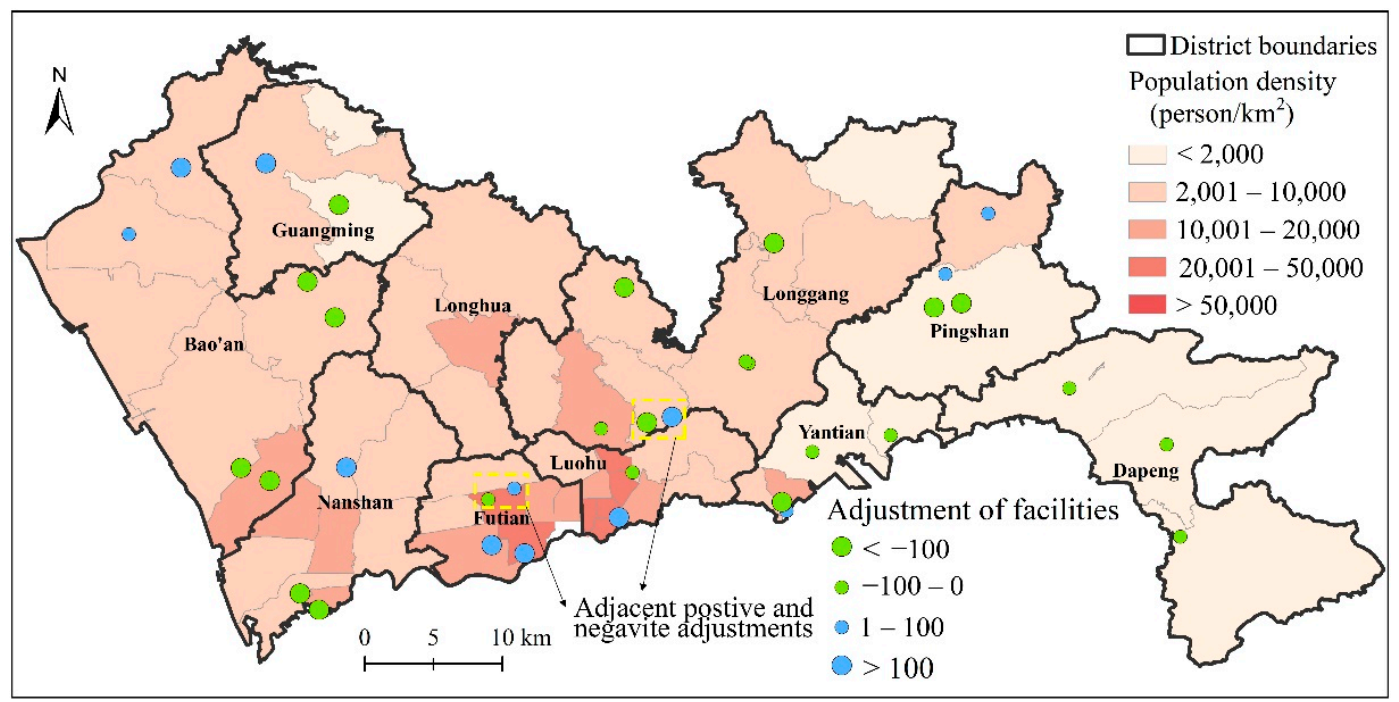

Figure 7. Size adjustments of existing secondary healthcare facilities according to optimization.

\subsection{Examining the Improvement of Accessibility Equality}

The disparities and distributions of the optimized as well as actual healthcare accessibility were further estimated and compared to examine whether and how the equality in accessibility is improved. The healthcare accessibility based on the actual distribution of healthcare facilities was estimated by using the Gaussian-based 2SFCA method with the same parameters as in the above optimization model. The disparity in accessibility was measured by coefficient of variation $(\mathrm{CV})$, which ranges from 0 to 1 , with a larger 
$\mathrm{CV}$ representing larger disparity. CV was selected as the measure of disparity because it can make the measures at two levels comparable. In the calculation of $\mathrm{CV}$, the standard deviation of accessibility is divided by the mean.

As shown in Table 3, the CVs of actual and optimized distributions of healthcare accessibility to tertiary facilities were 0.53 and 0.32 , respectively. Similarly, the respective CVs for actual and optimized secondary facilities were 0.58 and 0.36 . After optimization, the disparities in healthcare accessibility to tertiary and secondary facilities decreased by $40 \%$ and $38 \%$, respectively. In other words, the optimization improved the equality in the spatial accessibility to the tertiary and secondary healthcare facilities by $40 \%$ and $38 \%$, respectively.

Table 3. The coefficients of variation in actual and optimized healthcare accessibility.

\begin{tabular}{cccc}
\hline Facility Level & Actual & Optimized & Improvement \\
\hline Secondary facilities & 0.58 & 0.36 & $38 \%$ \\
Tertiary facilities & 0.53 & 0.32 & $40 \%$ \\
\hline
\end{tabular}

The accessibility was calculated first for discrete community locations, and then extrapolated into continuous distribution with the inverse distance weighted spatial interpolation method. As shown in Figure 8, after optimization, the healthcare accessibility to the tertiary facilities ranging from 0.0005 to 0.0010 is relatively evenly distributed in Shenzhen. The distribution of higher accessibility was found to be positively related to population density distribution. Low accessibility could only be observed in few marginal areas.

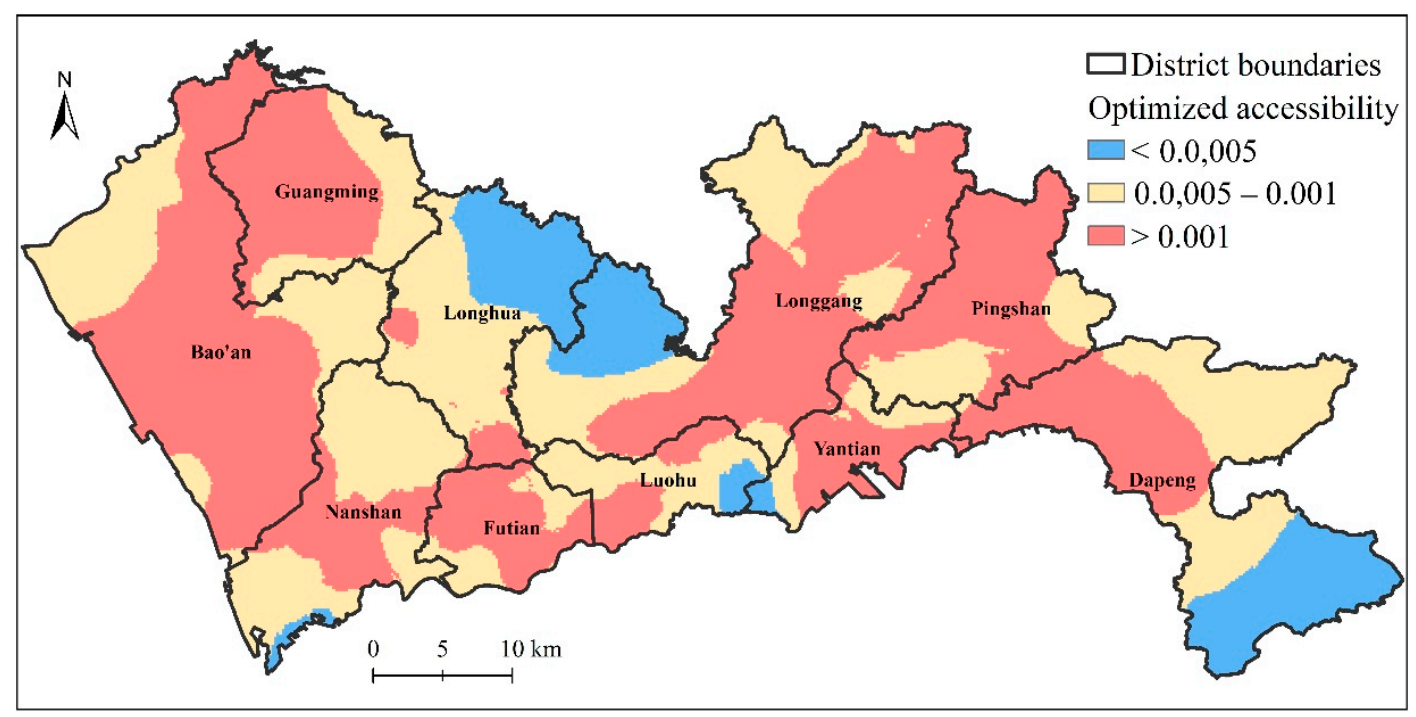

Figure 8. The distribution of optimized accessibility to tertiary healthcare facilities.

As shown in Figure 9, the optimized healthcare accessibility to the secondary facilities in most areas was found to range from 0.0004 to 0.008 in Shenzhen. However, the distributions of higher and lower accessibility were more dispersed than that of the tertiary facilities. This corresponds to the fact that the distribution of higher-level facilities is generally more concentrated. This proves that the optimization resulted in relatively equal healthcare accessibility to both the tertiary and secondary facilities. 


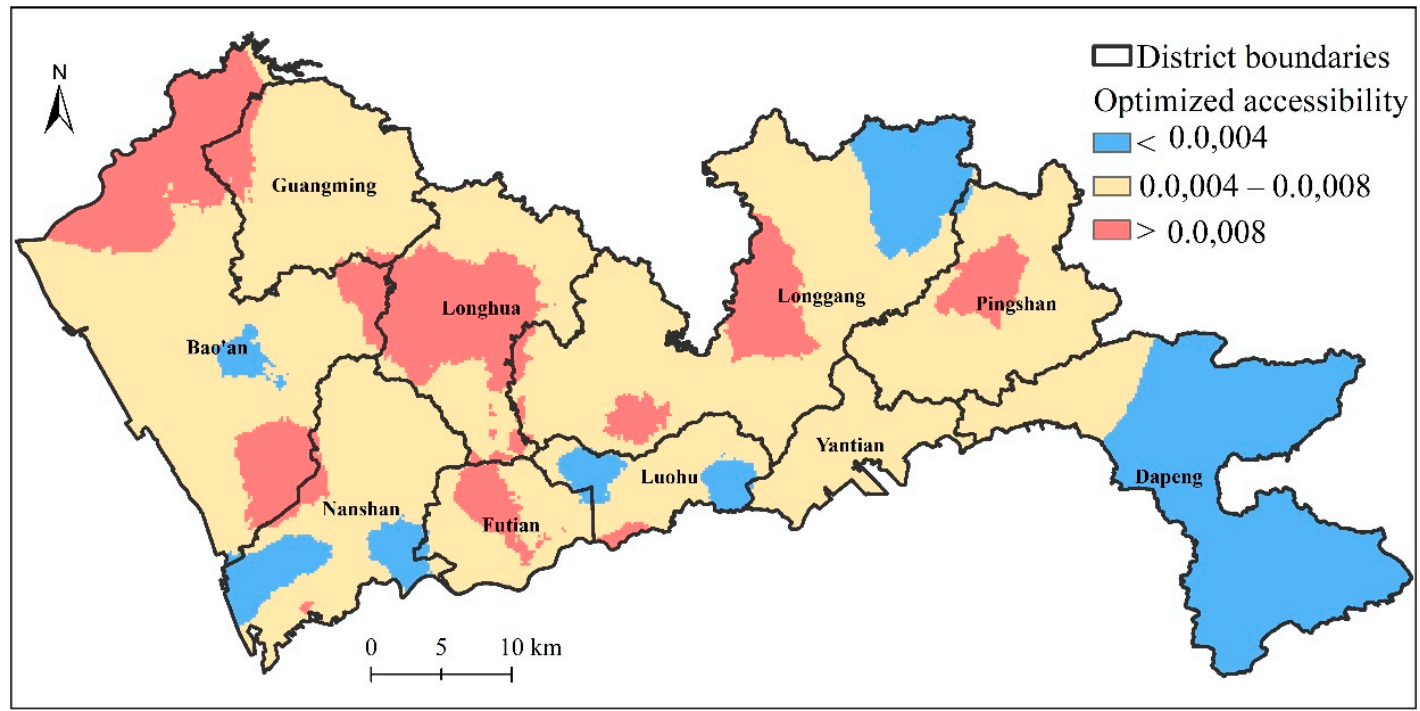

Figure 9. The distribution of optimized accessibility to secondary healthcare facilities.

\section{Discussion}

Rational distribution and equalization of healthcare services are critical for the improvement of health and well-being. Equality-oriented spatial optimization models can act as scientific tools for healthcare facilities planning and policymaking. This study developed a hierarchical maximal equality model that sought to maximize the equality in healthcare accessibility. The case study of Shenzhen proves that the model can significantly improve the equality in healthcare accessibility at each level as compared to the status quo. Considering the ubiquitous inequality in healthcare accessibility and the popularity of equalization of healthcare services as a key policy goal in different contexts, this study provides replicable procedures and methods for promoting the equalization of healthcare accessibility. Furthermore, the model can be applied or adapted for analyzing other hierarchical facilities (e.g., educational facilities and public parks) because the equal accessibility to various public services is a common public policy goal.

Compared to the original maximal accessibility equality model, the hierarchical maximal accessibility equality model developed in this study further incorporates hierarchical features of healthcare facilities. It specifies different catchment area sizes of, and distance friction effects for facilities at various levels. The empirical analyses demonstrate that the numbers and distribution of healthcare facilities at various levels are quite different. Existing studies have reported significant differences between healthcare accessibility at various levels [32-34]. The model developed in this study can better quantify the accessibility to hierarchical healthcare facilities across levels and optimize the equality of such accessibility. It highlights that the hierarchy structure of healthcare facilities should be carefully considered in the spatial optimization of public facilities.

Spatial optimization of hierarchical facilities is a classic and recurrent topic, as mentioned previously. A set of hierarchical location-allocation models have been developed in the past several decades [24,36,37]. These existing studies highlight the needs for taking into account the hierarchy structure of healthcare facilities in the optimization but fail to address the equality issue and comprehensively measure accessibility. This study contributes to the efforts in this respect by extending the maximal equality model into a hierarchical location-allocation model. Compared to existing hierarchical location-allocation models, it improves the measurement of accessibility, namely, interactions between demand and supply, by using a Gaussian-based 2SFCA method.

Furthermore, this study is one of the first studies that has attempted to account for both existing facility locations and newly added locations in the location-allocation analysis. The results reveal that not only existing facilities should be adjusted, but more 
new facilities need to be added in areas that are not well served by existing facilities. Considering that substantial fixed resources have been invested in existing facilities, such setting of candidate facility locations can better reflect the actual base of optimization or planning. Therefore, the procedures developed in this study can provide more feasible solutions of facility planning.

Our findings suggest a dispersal strategy to improve spatial equality in healthcare accessibility in Shenzhen by reallocating the existing healthcare resources and supplying extra resources. Generally, the sizes of existing facilities in central areas should be decreased, while the sizes of existing facilities should be increased, and more new facilities should be constructed in periphery areas. Considering that the space and land resources in central areas are in great shortage, the dispersal strategy is feasible to be put into implementation. However, it should be noted that our analyses are based on the population data (i.e., demand for healthcare resources) in 2010, and future growth of demands for healthcare resources were not considered. According to relevant plans in Shenzhen, peripheral areas are expected to experience larger population growth in the future. Therefore, the dispersal strategy can work even when the future population growth is taken into account. Note that the optimized healthcare facilities in the peripheral areas are middle-to-large-sized but dispersedly distributed. This suggests that the sizes of facilities in the peripheral areas should be expanded on one hand, and more new facilities may need to be built in these areas.

Despite the strengths of our study, there are also some limitations. First, the costs of the size adjustments of existing facilities are not considered in the optimization. As a result, in the optimized distribution of healthcare facilities, some facilities that need positive- or negative-size adjustments are close to each other. It is suggested that adjustments of existing facilities in such cases may be unnecessary and should not be implemented. By doing so, the costs of adjustments could be saved while the impacts on healthcare accessibility are negligible. In future study, such costs of size adjustments should be modelled into the optimization objectives or constraint conditions in a more normative way. Nevertheless, our optimization results can act as a scientific baseline for decision making. Second, although the analysis unit in this study (community) is already the smallest geographical area used by the local government, the analysis may still be faced with the modifiable area unit problem (MAUP), due to different areas and irregular shapes of the communities. More efforts are needed to examine whether and to what extent MAUP exists, as well as to figure out how to address MAUP in location-allocation modelling. Third, the accessibility to facilities and its equality was optimized independently for each level on the basis of the characteristics of the current hierarchical healthcare system in Shenzhen. However, this assumption may be inappropriate in some cases. In future works, efforts should be made to explore the interaction between various facility levels and to optimize the equality of the overall healthcare accessibility.

\section{Conclusions}

This study proposes a hierarchical maximal accessibility equality model for optimizing the locations of hierarchical healthcare facilities to improve the equality in accessibility to them. It extends the maximal accessibility equality model, which aims to minimize the variance of spatial accessibility to facilities by accounting for the hierarchical features of healthcare facilities. The minimal variance in spatial accessibility is pursued at each respective level. The Gaussian-based 2SFCA method is applied to measure the spatial accessibility to healthcare facilities at each level. The optimization model is solved by using the PSO algorithm. The empirical results demonstrate that healthcare facilities at each level need to be more dispersedly distributed to achieve maximal accessibility equality in Shenzhen. Compared to the current distribution, the accessibility equality of tertiary and secondary healthcare facilities in optimal solutions can be improved by $40 \%$ and $38 \%$, respectively, which proves the validity of the proposed optimization model. Both newly added facilities and adjustments of existing facilities are needed to achieve equal healthcare 
accessibility. Furthermore, the optimization results are quite different for facilities at different levels, which highlights the importance of considering hierarchy structure in the optimization of healthcare facilities. The findings provide evidence-based suggestions for the policymaking in Shenzhen to improve the accessibility to hierarchical healthcare facilities. All in all, this study provides transferable methods for the equality-oriented spatial optimization of hierarchical facilities.

Author Contributions: Conceptualization, Z.T., Q.W. and W.H.; methodology, Z.T.; software, Z.T.; validation, Z.T. and W.H.; formal analysis, Z.T.; investigation, Z.T., Q.W. and W.H.; resources, Z.T., Q.W. and W.H.; data curation, Z.T.; writing-original draft preparation, Z.T. and W.H.; writing—review and editing, Z.T., Q.W. and W.H.; visualization, Z.T.; project administration, Z.T.; funding acquisition, Z.T. All authors have read and agreed to the published version of the manuscript.

Funding: This work was funded by the National Natural Science Foundation of China (grant number 42101189) and the Fundamental Research Funds for the Central Universities. The funding bodies had no direct role in the design of the study or the collection, analysis, and interpretation of data, or in writing the manuscript.

Institutional Review Board Statement: Not applicable.

Informed Consent Statement: Not applicable.

Data Availability Statement: The healthcare facility data can be obtained from the website of Shenzhen Municipal Health Commission (http:/ / wjw.sz.gov.cn/bmfw/wycx/fwyl/yycx/index.html accessed on 26 October 2021). The travel time data were estimated using the public web API of Baidu Map (http:/ / lbsyun.baidu.com/index.php?title=jspopular/guide/routeplan accessed on 26 October 2021). The Particle Swarm Optimization Toolbox is available at https:/ /ww2.mathworks.cn/ matlabcentral/fileexchange/7506-particle-swarm-optimization-toolbox accessed on 26 October 2021.

Acknowledgments: We would like to thank the editors and anonymous reviewers for their insightful suggestions.

Conflicts of Interest: The authors declare no conflict of interest.

\section{References}

1. Corburn, J. Toward the Healthy City: People, Places, and the Politics of Urban Planning; The MIT Press: Cambridge, MA, USA, 2009.

2. Fujita, M.; Sato, Y.; Nagashima, K.; Takahashi, S.; Hata, A. Impact of geographic accessibility on utilization of the annual health check-ups by income level in Japan: A multilevel analysis. PLOS ONE 2017, 12, e0177091.

3. Onega, T.; Duell, E.J.; Shi, X.; Wang, D.; Demidenko, E.; Goodman, D. Geographic access to cancer care in the U.S. Cancer 2008, 112, 909-918. [CrossRef]

4. Wang, F. Measurement, optimization and impact of healthcare accessibility: A methodological review. Ann. Assoc. Am. Geogr. 2012, 102, 1104-1112. [CrossRef]

5. Khan, A.A. An integrated approach to measuring potential spatial access to health care services. Socioecon. Plan. Sci. 1992, 26, 275-287. [CrossRef]

6. Wang, F.; Luo, W. Assessing spatial and nonspatial factors for healthcare access: Towards an integrated approach to defining health professional shortage areas. Health Place 2005, 11, 131-146. [CrossRef]

7. Hansen, W.G. How Accessibility Shapes Land Use. J. Am. Inst. Plan. 1959, 25, 73-76. [CrossRef]

8. Falchetta, G.; Hammad, A.; Shayegh, S. Planning universal accessibility to public health care in sub-Saharan Africa. Proc. Natl. Acad. Sci. USA 2020, 117, 31760-31769. [CrossRef]

9. Weiss, D.J.; Nelson, A.; Vargas-Ruiz, C.A.; Gligorić, K.; Bavadekar, S.; Gabrilovich, E.; Bertozzi-Villa, A.; Rozier, J.; Gibson, H.S.; Shekel, T.; et al. Global maps of travel time to healthcare facilities. Nat. Med. 2020, 26, 1835-1838. [CrossRef] [PubMed]

10. The State Council of the People's Republic of China. The Guiding Opinions on Promoting the Construction of Hierarchical Medical System. 2015. Available online: http:/ / www.gov.cn/xinwen/2016-10/25/content_5124174.htm (accessed on 12 April 2020).

11. The State Council of the People's Republic of China. The Outline of the Healthy China 2030 Plan. 2016. Available online: http://www.gov.cn/zhengce/content/2015-09/11/content_10158.htm (accessed on 12 April 2020).

12. Kang, J.-Y.; Michels, A.; Lyu, F.; Wang, S.; Agbodo, N.; Freeman, V.L.; Wang, S. Rapidly measuring spatial accessibility of COVID-19 healthcare resources: A case study of Illinois, USA. Int. J. Health Geogr. 2020, 19, 36. [CrossRef]

13. Pereira, R.H.M.; Braga, C.K.V.; Servo, L.M.; Serra, B.; Amaral, P.; Gouveia, N.; Paez, A. Geographic access to COVID-19 healthcare in Brazil using a balanced float catchment area approach. Soc. Sci. Med. 2021, 273, 113773. [CrossRef]

14. McGrail, M.R.; Humphreys, J.S. Measuring spatial accessibility to primary health care services: Utilising dynamic catchment sizes. Appl. Geogr. 2014, 54, 182-188. [CrossRef] 
15. Polzin, P.; Borges, J.; Coelho, A.N. An extended kernel density two-step floating catchment area method to analyze access to health care. Environ. Plan. B Plan. Des. 2014, 41, 717-735. [CrossRef]

16. Tao, Z.; Liu, Z.; Cheng, Y. Hierarchical two-step floating catchment area (2SFCA) method: Measuring the spatial accessibility to hierarchical healthcare facilities in Shenzhen, China. Int. J. Equity Health 2020, 19, 164. [CrossRef]

17. Dai, D. Racial/ethnic and socioeconomic disparities in urban green space accessibility: Where to intervene? Landsc. Urban Plan 2011, 102, 234-244. [CrossRef]

18. Langford, M.; Higgs, G.; Fry, R. Multi-modal two-step floating catchment area analysis of primary health care accessibility. Health Place 2016, 38, 70-81. [CrossRef]

19. Tao, Z.; Cheng, Y. Modelling the spatial accessibility of the elderly to healthcare services in Beijing, China. Environ. Plan. B Urban Anal. City Sci. 2019, 46, 1132-1147. [CrossRef]

20. Neutens, T. Accessibility, equity and health care: Review and research directions for transport geographers. J. Transp. Geogr. 2015, 43, 14-27. [CrossRef]

21. Cheng, G.; Zeng, X.; Duan, L.; Lu, X.; Sun, H.; Jiang, T.; Li, Y. Spatial difference analysis for accessibility to high level hospitals based on travel time in Shenzhen, China. Habitat Int. 2016, 53, 485-494. [CrossRef]

22. Drezner, Z.; Hamacher, H.W. Facility Location: Applications and Theory; Springer: New York, NY, USA, 2002.

23. Tong, D.; Murray, A.T. Spatial Optimization in Geography. Ann. Assoc. Am. Geogr. 2012, 102, 1290-1309. [CrossRef]

24. Owen, S.H.; Daskin, M.S. Strategic facility location: A review. Eur. J. Oper. Res. 1998, 111, 423-447. [CrossRef]

25. Kontodimopoulos, N.; Nanos, P.; Niakas, D. Balancing efficiency of health services and equity of access in remote areas in Greece. Health Policy 2006, 76, 49-57. [CrossRef]

26. Church, R.L. Location modelling and GIS. In Geographical Information Systems; Longley, P.A., Goodchild, M., Maguire, D., Rhind, D., Eds.; John Wiley: New York, NY, USA, 1999; pp. 293-303.

27. Murray, A.T. Maximal Coverage Location Problem. Int. Reg. Sci. Rev. 2015, 39, 5-27. [CrossRef]

28. Wang, F.; Tang, Q. Planning toward equal accessibility to services: A quadratic programming approach. Environ. Plan. B Plan. Des. 2013, 40, 195-212. [CrossRef]

29. Tao, Z.; Cheng, Y.; Dai, T.; Rosenberg, M.W. Spatial optimization of residential care facility locations in Beijing, China: Maximum equity in accessibility. Int. J. Health Geogr. 2014, 13, 33. [CrossRef]

30. Luo, J.; Tian, L.; Luo, L.; Yi, H.; Wang, F. Two-Step Optimization for Spatial Accessibility Improvement: A Case Study of Health Care Planning in Rural China. BioMed Res. Int. 2017, 2017, 1-12. [CrossRef] [PubMed]

31. Dai, T.; Liu, Z.; Liao, C.; Cai, H. Toward Equal Opportunity of Primary Education: Introducing a Lottery into China's ProximityBased Enrollment System. Prof. Geogr. 2019, 71, 210-220. [CrossRef]

32. Jin, M.; Liu, L.; Tong, D.; Gong, Y.; Liu, Y. Evaluating the Spatial Accessibility and Distribution Balance of Multi-Level Medical Service Facilities. Int. J. Environ. Res. Public Health 2019, 16, 1150. [CrossRef]

33. Zhang, S.; Song, X.; Wei, Y.; Deng, W. Spatial Equity of Multilevel Healthcare in the Metropolis of Chengdu, China: A New Assessment Approach. Int. J. Environ. Res. Public Health 2019, 16, 493. [CrossRef] [PubMed]

34. Ma, X.; Ren, F.; Du, Q.; Liu, P.; Li, L.; Xi, Y.; Jia, P. Incorporating multiple travel modes into a floating catchment area framework to analyse patterns of accessibility to hierarchical healthcare facilities. J. Transp. Health 2019, 15, 100675. [CrossRef]

35. Hodgson, M.J. Alternative Approaches to Hierarchical Location-Allocation Systems. Geogr. Anal. 1984, 16, 275-281. [CrossRef]

36. Şahin, G.; Süral, H. A review of hierarchical facility location models. Comput. Oper. Res. 2007, 34, 2310-2331. [CrossRef]

37. Farahani, R.Z.; Hekmatfar, M.; Fahimnia, B.; Kazemzadeh, N. Hierarchical facility location problem: Models, classifications, techniques, and applications. Comput. Ind. Eng. 2014, 68, 104-117. [CrossRef]

38. García-Palomares, J.C.; Gutiérrez, J.; Latorre, M. Optimizing the location of stations in bike-sharing programs: A GIS approach. Appl. Geogr. 2012, 35, 235-246. [CrossRef]

39. Drezner, T.; Drezner, Z. The gravity p-median model. Eur. J. Oper. Res. 2007, 179, 1239-1251. [CrossRef]

40. Narula, S.C. Hierarchical location-allocation problems: A classification scheme. Eur. J. Oper. Res. 1984, 15, 93-99. [CrossRef]

41. Espejo, L.G.A.; Galv, O.R.D.; Boffey, B. Dual-based heuristics for a hierarchical covering location problem. Comput. Oper. Res. 2003, 30, 165-180. [CrossRef]

42. Teixeira, J.C.; Antunes, A.P. A hierarchical location model for public facility planning. Eur. J. Oper. Res. 2008, 185, 92-104. [CrossRef]

43. Wang, F.; Fu, C.; Shi, X. Planning towards maximum equality in accessibility to NCI cancer centers in the U.S. In Spatial Analysis in Health Geography; Kanaroglou, P., Delmelle, E., Ghosh, D., Paez, A., Eds.; Ashgate Publishing: Farnham, UK, 2015; pp. 261-274.

44. Li, X.; Wang, F.; Yi, H. A two-step approach to planning new facilities towards equal accessibility. Environ. Plan. B Urban Anal. City Sci. 2017, 44, 994-1011. [CrossRef]

45. Shenzhen Municipal Health Commission. List of Healthcare Facilities in Shenzhen. 2020. Available online: http://wjw.sz.gov.cn/ bmfw/wycx/fwyl/yycx/index.html (accessed on 9 October 2020).

46. Baidu Map. Development Document of Baidu Map Route Plan Web API. 2020. Available online: http://lbsyun.baidu.com/index. php?title=jspopular $/$ guide / routeplan (accessed on 9 October 2020).

47. Wang, F.; Xu, Y. Estimating O-D travel time matrix by Google Maps API: Implementation, advantages, and implications. Ann. GIS 2011, 17, 199-209. [CrossRef] 
48. Dony, C.C.; Delmelle, E.M.; Delmelle, E.C. Re-conceptualizing accessibility to parks in multi-modal cities: A Variable-width Floating Catchment Area (VFCA) method. Landsc. Urban Plan. 2015, 143, 90-99. [CrossRef]

49. Chu, H.-J.; Lin, B.-C.; Yu, M.-R.; Chan, T.-C. Minimizing Spatial Variability of Healthcare Spatial Accessibility-The Case of a Dengue Fever Outbreak. Int. J. Environ. Res. Public Health 2016, 13, 1235. [CrossRef] [PubMed]

50. Kennedy, J.; Eberhart, R.C. Particle swarm optimization. In Proceedings of the ICNN'95-International Conference on Neural Networks, Perth, WA, Australia, 27 November-1 December 1995.

51. Birge, B. Particle Swarm Optimization Toolbox. 2006. Available online: https://ww2.mathworks.cn/matlabcentral/fileexchange/ 7506-particle-swarm-optimization-toolbox (accessed on 5 October 2020). 